\title{
On an inverse problem for a fractional semilinear elliptic equation involving a magnetic potential
}

\author{
$\mathrm{Li} \mathrm{Li}$ \\ Department of Mathematics, University of Washington, \\ Seattle, WA 98195, USA
}

\begin{abstract}
We study a class of fractional semilinear elliptic equations and formulate the corresponding Calderón problem. We determine the nonlinearity from the exterior partial measurements of the Dirichlet-to-Neumann map by using first order linearization and the Runge approximation property.
\end{abstract}

\section{Introduction}

The study of the fractional Calderón problem was initiated in [10 where the authors considered an inverse problem for the fractional linear operator

$$
(-\Delta)^{s}+q \quad(0<s<1) .
$$

See 1, 2, 3, 4, 8, 9, 23, for further studies based on 10.

Recently a fractional semilinear Calderón problem has been studied in [18. This inverse problem can be viewed as a nonlocal analogue of the classical semilinear Calderón problem studied in 13 . In [18, the authors considered the exterior Dirichlet problem

$$
(-\Delta)^{s} u+a(x, u)=0 \text { in } \Omega,\left.\quad u\right|_{\Omega_{e}}=g
$$

where $\Omega$ is a bounded domain with $C^{1,1}$ boundary and $\Omega_{e}:=\mathbb{R}^{n} \backslash \bar{\Omega}, n \geq 2$. Under some regularity assumptions on $a(\cdot, \cdot)$, the authors proved that the nonlinearity $a(\cdot, \cdot)$ can be uniquely determined from the exterior partial measurements of the Dirichlet-to-Neumann map

$$
\Lambda_{a}:\left.g \rightarrow(-\Delta)^{s} u_{g}\right|_{\Omega_{e}} .
$$

In this paper, we extend the earlier result in [18. We study the generalized operator $\mathcal{R}_{A}^{s}$, which is formally defined by

$$
\mathcal{R}_{A}^{s} u(x):=2 \lim _{\epsilon \rightarrow 0^{+}} \int_{\mathbb{R}^{n} \backslash B_{\epsilon}(x)}\left(u(x)-R_{A}(x, y) u(y)\right) K(x, y) d y
$$

where $K(x, y)=c_{n, s} /|x-y|^{n+2 s}, A$ is a fixed real vector-valued magnetic potential and

$$
R_{A}(x, y):=\cos \left((x-y) \cdot A\left(\frac{x+y}{2}\right)\right)
$$


Clearly $\mathcal{R}_{A}^{s}$ coincides with $(-\Delta)^{s}$ when $A=0$ and $\mathcal{R}_{A}^{s}$ is the real part of the fractional magnetic Laplacian $(-\Delta)_{A}^{s}$ formally defined by

$$
(-\Delta)_{A}^{s} u(x):=2 \lim _{\epsilon \rightarrow 0^{+}} \int_{\mathbb{R}^{n} \backslash B_{\epsilon}(x)}\left(u(x)-e^{i(x-y) \cdot A\left(\frac{x+y}{2}\right)} u(y)\right) K(x, y) d y
$$

(see for instance, [5, 24]) when $u$ is real-valued. We study the exterior Dirichlet problem

$$
\mathcal{R}_{A}^{s} u+a(x, u)=0 \text { in } \Omega,\left.\quad u\right|_{\Omega_{e}}=g .
$$

Our goal is to determine the nonlinearity $a(\cdot, \cdot)$ from the knowledge of the associated Dirichletto-Neumann map. Our inverse problem can be viewed as a semilinear analogue of the fractional Calderón problem studied in [20], which is a nonlocal analogue of the classical Calderón problem for the magnetic Laplacian studied in [7, 14, 21, 25].

Linearization is a standard technique used in solving the nonlinear Calderón problem. See for instance, [12, 26, 27]. In [18], the authors applied high order linearization to prove their uniqueness theorem (see Section 3). See 15, 16, 19, for similar techniques used in solving the semilinear Calderón problem for local operators. In this paper, we use the first order linearization in the Sobolev space $H^{s}\left(\mathbb{R}^{n}\right)$ and the Runge approximation property obtained in [20] instead to prove our uniqueness theorem.

To ensure that the exterior Dirichlet problem (3) is well-posed for small $g$, we assume that $A$ satisfies some boundedness condition and the nonlinearity $a(x, z): \Omega \times \mathbb{R} \rightarrow \mathbb{R}$ satisfies

(i) $z \rightarrow a(\cdot, z)$ is analytic with values in $C^{s}(\Omega)$;

(ii) $a(x, 0)=0$ and $\partial_{z} a(x, 0) \geq c>0$ for some constant $c>0$

so we have the Taylor's expansion

$$
a(x, z)=\sum_{k=1}^{\infty} a_{k}(x) \frac{z^{k}}{k !}, \quad a_{k}(x)=\partial_{z}^{k} a(x, 0) \in C^{s}(\Omega)
$$

where the series converges in the Hölder space $C^{s}(\Omega)$ topology.

Under the assumptions above, we can define the bounded solution operator $Q_{A, a}: g \rightarrow u_{g}$ and the Dirichlet-to-Neumann map $\Lambda_{A, a}$ formally given by

$$
\Lambda_{A, a} g:=\left.\mathcal{R}_{A}^{s}\left(Q_{A, a}(g)\right)\right|_{\Omega_{e}}
$$

to formulate corresponding Calderón problem.

The following theorem is the main result in this paper.

Theorem 1.1. Let $\Omega \subset \mathbb{R}^{n}, n \geq 2$ be a bounded domain with $C^{1,1}$ boundary. Suppose $\Omega \cup \operatorname{supp} A \subset$ $B_{r}(0)$ for some constant $r>0$ and $\|A\|_{L^{\infty}\left(\mathbb{R}^{n}\right)} \leq \pi /(8 \sqrt{n} r)$, $a^{(j)}$ satisfy (i) and (ii), $W_{j}$ are open sets s.t. $W_{j} \cap B_{3 r}(0)=\emptyset(j=1,2)$. If

$$
\left.\Lambda_{A, a^{(1)}} g\right|_{W_{2}}=\left.\Lambda_{A, a^{(2)}} g\right|_{W_{2}}, \quad g \in C_{c}^{\infty}\left(W_{1}\right)
$$

whenever $\|g\|_{C^{2}\left(\mathbb{R}^{n}\right)}$ is sufficiently small, then $a^{(1)}=a^{(2)}$ in $\Omega \times \mathbb{R}$.

Remark. The nonlinear problem is reduced to the linear one when $a^{(j)}(x, z)=a_{1}^{(j)}(x) z(j=1,2)$. If this is the case, then the statement still holds after we replace $\|A\|_{L^{\infty}\left(\mathbb{R}^{n}\right)} \leq \pi /(8 \sqrt{n} r)$ by the weakened assumption $A \in L^{\infty}\left(\mathbb{R}^{n}\right)$. See Theorem 1.1 in [20]. 
The rest of this paper is organized in the following way. In Section 2, we summarize the background knowledge. We prove that the nonlinear problem (3) is well-posed in Section 4, based on the $L^{\infty}$ estimate and the Hölder regularity theorem for the corresponding linear problem proved in Section 3. In Section 5, we prove the main theorem.

Acknowledgment. The author is partly supported by National Science Foundation and would like to thank Professor Gunther Uhlmann for helpful discussions.

\section{Preliminaries}

Throughout this paper

- $n \geq 2$ denotes the space dimension and $0<s<1$ denotes the fractional power

- $\Omega$ denotes a bounded domain with $C^{1,1}$ boundary and $\Omega_{e}:=\mathbb{R}^{n} \backslash \bar{\Omega}$

- $B_{r}(0)$ denotes the open ball centered at the origin with radius $r>0$ and $\overline{B_{r}}(0)$ denotes the closure of $B_{r}(0)$

- $A: \mathbb{R}^{n} \rightarrow \mathbb{R}^{n}$ denotes a real vector-valued magnetic potential

- $c, C, C^{\prime}, C_{1}, \cdots$ denote positive constants (which may depend on some parameters but always independent of small constants $\epsilon, \rho$ )

- $\int \cdots \int=\int_{\mathbb{R}^{n}} \cdots \int_{\mathbb{R}^{n}}$

- $X^{*}$ denotes the continuous dual space of $X$ and write $\langle f, u\rangle=f(u)$ for $u \in X, f \in X^{*}$

- $\|\cdot\|_{C^{2}\left(\mathbb{R}^{n}\right)}$ is defined by

$$
\|f\|_{C^{2}\left(\mathbb{R}^{n}\right)}=\sum_{|\alpha| \leq 2}\left\|\partial^{\alpha} f\right\|_{L^{\infty}\left(\mathbb{R}^{n}\right)} .
$$

\section{$2.1 \quad$ Function Spaces}

Throughout this paper we refer all function spaces to real-valued function spaces.

For $t \in \mathbb{R}$, we have Sobolev spaces

$$
H^{t}\left(\mathbb{R}^{n}\right):=\left\{u \in \mathcal{S}^{\prime}\left(\mathbb{R}^{n}\right): \int\left(1+|\xi|^{2}\right)^{t}|\mathcal{F} u(\xi)|^{2} d \xi<\infty\right\}
$$

where $\mathcal{F}$ is the Fourier transform and $\mathcal{S}^{\prime}\left(\mathbb{R}^{n}\right)$ is the space of temperate distributions. We have the natural identification $H^{-t}\left(\mathbb{R}^{n}\right)=H^{t}\left(\mathbb{R}^{n}\right)^{*}$. Let $U$ be an open set and $F$ be a closed set in $\mathbb{R}^{n}$,

$$
\begin{gathered}
H^{t}(U):=\left\{\left.u\right|_{U}: u \in H^{t}\left(\mathbb{R}^{n}\right)\right\}, \quad H_{F}^{t}\left(\mathbb{R}^{n}\right):=\left\{u \in H^{t}\left(\mathbb{R}^{n}\right): \operatorname{supp} u \subset F\right\}, \\
\tilde{H}^{t}(U):=\text { the closure of } C_{c}^{\infty}(U) \text { in } H^{t}\left(\mathbb{R}^{n}\right) .
\end{gathered}
$$

Since $\Omega$ is a bounded domain with $C^{1,1}$ boundary implies $\Omega$ is Lipschitz bounded, then

$$
\tilde{H}^{t}(\Omega)=H_{\bar{\Omega}}^{t}\left(\mathbb{R}^{n}\right) .
$$


For $0<s<1$, one of the equivalent forms of the norm $\|\cdot\|_{H^{s}\left(\mathbb{R}^{n}\right)}$ is

$$
\|u\|_{H^{s}\left(\mathbb{R}^{n}\right)}:=\left(\|u\|_{L^{2}\left(\mathbb{R}^{n}\right)}^{2}+\iint \frac{|u(x)-u(y)|^{2}}{|x-y|^{n+2 s}} d x d y\right)^{1 / 2} .
$$

We have the Hölder space $C^{s}(U):=C^{0, s}(U)$ equipped with the standard norm given by

$$
\|f\|_{C^{s}(U)}:=\|f\|_{L^{\infty}(U)}+\sup _{x \neq y, x, y \in U} \frac{|f(x)-f(y)|}{|x-y|^{s}} .
$$

\subsection{The Operator $\mathcal{R}_{A}^{s}$}

In Section 1 we gave the formal pointwise definition of $\mathcal{R}_{A}^{s}$ in (1). Now we do a formal computation to motivate the bilinear form definition of $\mathcal{R}_{A}^{s}$.

It is clear from (2) that $R_{A}(x, y)=R_{A}(y, x)$. Hence for real-valued $u, v$, we can formally compute that

$$
\begin{gathered}
2 \iint_{\{|x-y| \geq \epsilon\}}\left(u(x)-R_{A}(x, y) u(y)\right) v(x) K(x, y) d y d x \\
=\iint_{\{|x-y| \geq \epsilon\}}\left[\left(u(x)-R_{A}(x, y) u(y)\right) v(x) K(x, y)+\left(u(y)-R_{A}(x, y) u(x)\right) v(y) K(x, y)\right] d y d x \\
=\operatorname{Re} \iint_{\{|x-y| \geq \epsilon\}}\left(u(x)-e^{i(x-y) \cdot A\left(\frac{x+y}{2}\right)} u(y)\right)\left(v(x)-e^{-i(x-y) \cdot A\left(\frac{x+y}{2}\right)} v(y)\right) K(x, y) d x d y .
\end{gathered}
$$

Now let $\epsilon \rightarrow 0^{+}$.

Definition 2.1. For real-valued $u, v$, we define $\mathcal{R}_{A}^{s}$ by the bilinear form

$$
\begin{gathered}
\left\langle\mathcal{R}_{A}^{s} u, v\right\rangle:=\operatorname{Re} \iint\left(u(x)-e^{i(x-y) \cdot A\left(\frac{x+y}{2}\right)} u(y)\right)\left(v(x)-e^{-i(x-y) \cdot A\left(\frac{x+y}{2}\right)} v(y)\right) K(x, y) d x d y \\
=2 \iint\left(u(x)-R_{A}(x, y) u(y)\right) v(x) K(x, y) d x d y
\end{gathered}
$$

It is easy to verify that

$$
\left\langle\mathcal{R}_{A}^{s} u, v\right\rangle=\left\langle\mathcal{R}_{A}^{s} v, u\right\rangle .
$$

Definition 2.2. We define the magnetic Sobolev norm $\|\cdot\|_{H_{A}^{s}}$ by

$$
\|u\|_{H_{A}^{s}}:=\left(\|u\|_{L^{2}}^{2}+[u]_{H_{A}^{s}}^{2}\right)^{1 / 2}
$$

where $[u]_{H_{A}^{s}}:=\left\langle\mathcal{R}_{A}^{s} u, u\right\rangle^{\frac{1}{2}}$.

This norm was introduced in [5, 24]. As we mentioned in Section $1, \mathcal{R}_{A}^{s}$ is the real part of the fractional magnetic Laplacian, whose properties have been studied in [20. In fact, Lemma 3.3 and Proposition 3.4 in [20] imply the following proposition. 
Proposition 2.3. Suppose $0<s<1$ and $A \in L^{\infty}\left(\mathbb{R}^{n}\right)$, then we have the norm equivalence $\|\cdot\|_{H_{A}^{s}} \sim\|\cdot\|_{H^{s}}$ and the operator

$$
\mathcal{R}_{A}^{s}: H^{s}\left(\mathbb{R}^{n}\right) \rightarrow H^{-s}\left(\mathbb{R}^{n}\right)
$$

is linear and bounded.

From now on we always assume $A \in L^{\infty}\left(\mathbb{R}^{n}\right)$. Corollary 5.3 in 20] implies the following proposition, which will be used later in the proof of the main theorem.

Proposition 2.4. Suppose $\Omega \cup \operatorname{supp} A \subset B_{r}(0)$ for some $r>0, W$ is an open set s.t. $W \backslash \overline{B_{3 r}}(0) \neq \emptyset$. If

$$
u \in \tilde{H}^{s}(\Omega),\left.\quad \mathcal{R}_{A}^{s} u\right|_{W}=0
$$

then $u=0$ in $\mathbb{R}^{n}$.

\section{The Linear Exterior Problem}

Throughout this section we assume $0<c \leq q(x) \in L^{\infty}(\Omega)$.

We first recall some results in [20. Based on Proposition 4.3 and Proposition 4.5 in [20], we have the following proposition.

Proposition 3.1. The bilinear form

$$
B_{A, q}(u, v):=\left\langle\mathcal{R}_{A}^{s} u, v\right\rangle+\int_{\Omega} q u v
$$

is coercive and bounded on $\tilde{H}^{s}(\Omega) \times \tilde{H}^{s}(\Omega)$. The exterior problem

$$
\left\{\begin{aligned}
\left(\mathcal{R}_{A}^{s}+q\right) u=0 & \text { in } \Omega \\
u=g & \text { in } \Omega_{e}
\end{aligned}\right.
$$

has a unique (weak) solution $u_{g} \in H^{s}\left(\mathbb{R}^{n}\right)$ for each $g \in H^{s}\left(\mathbb{R}^{n}\right)$ and the solution operator

$$
P_{A, q}: g \rightarrow u_{g}
$$

is bounded on $H^{s}\left(\mathbb{R}^{n}\right)$.

Proposition 5.4 in [20] implies the Runge approximation property of $\mathcal{R}_{A}^{s}+q$, which will be used later in the proof of the main theorem.

Proposition 3.2. Suppose $\Omega \cup \operatorname{supp} A \subset B_{r}(0)$ for some $r>0, W$ is an open set s.t. $W \subset \Omega_{e}$ and $W \backslash \overline{B_{3 r}}(0) \neq \emptyset$, then

$$
S:=\left\{\left.P_{A, q} f\right|_{\Omega}: f \in C_{c}^{\infty}(W)\right\}
$$

is dense in $L^{2}(\Omega)$.

Next we prove an $L^{\infty}$ estimate and a Hölder regularity theorem, which will be useful in later sections when we deal with the nonlinear problem. 


\section{$3.1 L^{\infty}$ Estimate}

Lemma 3.3. If $g \in C_{c}^{\infty}\left(\mathbb{R}^{n}\right)$, then $(-\Delta)^{s} g \in L^{\infty}\left(\mathbb{R}^{n}\right)$ and

$$
\left\|(-\Delta)^{s} g\right\|_{L^{\infty}\left(\mathbb{R}^{n}\right)} \leq C\|g\|_{C^{2}\left(\mathbb{R}^{n}\right)}
$$

Proof. For $g \in C_{c}^{\infty}\left(\mathbb{R}^{n}\right)$, we have

$$
(-\Delta)^{s} g(x)=c_{n, s} \int \frac{2 g(x)-g(x+y)-g(x-y)}{|y|^{n+2 s}} d y
$$

(see for instance, Lemma 3.2 in [6]) so by using Taylor's expansion, we have

$$
\left|(-\Delta)^{s} g(x)\right| \leq c_{n, s}\left(\int_{|y| \leq 1}+\int_{|y|>1}\right) \frac{|2 g(x)-g(x+y)-g(x-y)|}{|y|^{n+2 s}} d y \leq C\|g\|_{C^{2}\left(\mathbb{R}^{n}\right)} .
$$

Lemma 3.4. If $A, g \in L^{\infty}\left(\mathbb{R}^{n}\right)$, then $\left((-\Delta)^{s}-\mathcal{R}_{A}^{s}\right) g \in L^{\infty}\left(\mathbb{R}^{n}\right)$ and

$$
\left\|\left((-\Delta)^{s}-\mathcal{R}_{A}^{s}\right) g\right\|_{L^{\infty}\left(\mathbb{R}^{n}\right)} \leq C\|g\|_{L^{\infty}\left(\mathbb{R}^{n}\right)} .
$$

Proof. Note that

$$
0 \leq 1-R_{A}(x, y)=2 \sin ^{2}\left(\frac{1}{2}(x-y) \cdot A\left(\frac{x+y}{2}\right)\right) \leq C_{A} \min \left\{1,|x-y|^{2}\right\}
$$

so we have

$$
\begin{gathered}
\left|\left((-\Delta)^{s}-\mathcal{R}_{A}^{s}\right) g(x)\right| \leq \int\left(1-R_{A}(x, y)\right) K(x, y)|g(y)| d y \\
=\left(\int_{|y-x| \leq 1}+\int_{|y-x|>1}\right)\left(1-R_{A}(x, y)\right) K(x, y)|g(y)| d y \leq C\|g\|_{L^{\infty}\left(\mathbb{R}^{n}\right)} .
\end{gathered}
$$

From now on we always assume $\Omega \cup \operatorname{supp} A \subset B_{r}(0)$ for some constant $r>0$ and $\|A\|_{L^{\infty}\left(\mathbb{R}^{n}\right)} \leq$ $\pi /(8 \sqrt{n} r)$. This coincides with the assumption on $A$ in the statement of Theorem 1.1.

Note that under this assumption, we have

$$
0 \leq R_{A}(x, y) \leq 1, \quad(x, y) \in\left(\mathbb{R}^{n} \times \mathbb{R}^{n}\right) \backslash\left(\Omega_{e} \times \Omega_{e}\right) .
$$

In fact, if $(x, y) \in \Omega \times \mathbb{R}^{n}$, then

(i) $y \in B_{3 r}(0)$, which implies $|x-y| \leq 4 r,\left|(x-y) \cdot A\left(\frac{x+y}{2}\right)\right| \leq \frac{\pi}{2}$;

(ii) $y \notin B_{3 r}(0)$, which implies $\left|\frac{x+y}{2}\right| \geq r, R_{A}=1$.

By symmetry of $R_{A}$, we know the claim also holds for $(x, y) \in \mathbb{R}^{n} \times \Omega$.

The following two propositions generalize Proposition 3.1 and Proposition 3.3 in [17].

Proposition 3.5. Suppose $0<c \leq q(x) \in L^{\infty}(\Omega)$. If $u \in H^{s}\left(\mathbb{R}^{n}\right)$ solves the exterior problem

$$
\left\{\begin{aligned}
\left(\mathcal{R}_{A}^{s}+q\right) u & =f & & \text { in } \Omega \\
u & =g & & \text { in } \Omega_{e}
\end{aligned}\right.
$$

for $0 \leq f \in H^{-s}(\Omega)$ and $0 \leq\left. g\right|_{\Omega_{e}} \in L^{\infty}\left(\Omega_{e}\right)$, then $u \geq 0$. 
Proof. Write $u=u^{+}-u^{-}$where $u^{+}=\max \{u, 0\}$ and $u^{-}=\max \{-u, 0\}$. Note that

$$
\left|u^{+}(x)-u^{+}(y)\right|+\left|u^{-}(x)-u^{-}(y)\right|=|u(x)-u(y)|
$$

so $u^{+}, u^{-} \in H^{s}\left(\mathbb{R}^{n}\right)$ and $\left.g\right|_{\Omega_{e}} \geq 0$ implies $u^{-} \in \tilde{H}^{s}(\Omega)$, so we have

$$
\left\langle\mathcal{R}_{A}^{s} u, u^{-}\right\rangle+\int_{\Omega} q u u^{-}=f\left(u^{-}\right) .
$$

Now write

$$
\begin{gathered}
\left\langle\mathcal{R}_{A}^{s} u, u^{-}\right\rangle=2 \iint\left(u(x)-R_{A}(x, y) u(y)\right) u^{-}(x) K(x, y) d x d y \\
=2\left(\int_{\Omega} \int_{\Omega}+\int_{\Omega_{e}} \int_{\Omega}\right)\left(u(x)-R_{A}(x, y) u(y)\right) u^{-}(x) K(x, y) d x d y=: I_{1}+I_{2} .
\end{gathered}
$$

Since $u^{-} u \leq 0$, then we have

$$
I_{2}=2 \int_{\Omega_{e}} \int_{\Omega}\left(u(x)-R_{A}(x, y) g(y)\right) u^{-}(x) K(x, y) d x d y \leq 0, \quad \int_{\Omega} q u u^{-} \leq 0 .
$$

Note that

$$
\begin{gathered}
I_{1}=2 \int_{\Omega} \int_{\Omega}\left(u(x)-R_{A}(x, y) u(y)\right) u^{-}(x) K(x, y) d x d y \\
=2 \int_{\Omega} \int_{\Omega}\left[\left(u^{+}(x)-R_{A}(x, y) u^{+}(y)\right) u^{-}(x)-\left(u^{-}(x)-R_{A}(x, y) u^{-}(y)\right) u^{-}(x)\right] K(x, y) d x d y \\
=-2 \int_{\Omega} \int_{\Omega}\left[R_{A}(x, y) u^{+}(y) u^{-}(x)+\left(u^{-}(x)-R_{A}(x, y) u^{-}(y)\right) u^{-}(x)\right] K(x, y) d x d y \\
\leq-2 \int_{\Omega} \int_{\Omega}\left(u^{-}(x)-R_{A}(x, y) u^{-}(y)\right) u^{-}(x) K(x, y) d x d y \\
=-\int_{\Omega} \int_{\Omega}\left[\left(u^{-}(x)-R_{A}(x, y) u^{-}(y)\right) u^{-}(x)+\left(u^{-}(y)-R_{A}(x, y) u^{-}(x)\right) u^{-}(y)\right] K(x, y) d x d y \\
=-\int_{\Omega} \int_{\Omega}\left(\left|u^{-}(x)\right|^{2}+\left|u^{-}(y)\right|^{2}-2 R_{A}(x, y) u^{-}(x) u^{-}(y)\right) K(x, y) d x d y \\
\leq-\int_{\Omega} \int_{\Omega}\left|u^{-}(x)-u^{-}(y)\right|^{2} K(x, y) d x d y .
\end{gathered}
$$

Since $f\left(u^{-}\right) \geq 0$, then the only possibility is

$$
\int_{\Omega} \int_{\Omega}\left|u^{-}(x)-u^{-}(y)\right|^{2} K(x, y) d x d y=0,
$$

which implies $u^{-}$is a non-negative constant $c_{0}$ in $\Omega$. Now we show $c_{0}$ has to be 0 .

Otherwise $u=-c_{0}<0$ in $\Omega$. In this case, for $x \in \Omega$, by pointwise definition we have

$$
\mathcal{R}_{A}^{s} u(x)=2 \lim _{\epsilon \rightarrow 0^{+}}\left(\int_{\Omega \backslash B_{\epsilon}(x)}+\int_{\Omega_{e}}\right)\left(u(x)-R_{A}(x, y) u(y)\right) K(x, y) d y
$$




$$
=2 \int_{\Omega}\left(1-R_{A}(x, y)\right)\left(-c_{0}\right) K(x, y) d y+2 \int_{\Omega_{e}}\left(-c_{0}-R_{A}(x, y) g(y)\right) K(x, y) d y \leq 0 .
$$

Both integrals converge since $\left.g\right|_{\Omega_{e}} \in L^{\infty}\left(\Omega_{e}\right)$ and $0 \leq 1-R_{A}(x, y) \leq C_{A}|x-y|^{2}$. Now we have got the contradiction

$$
f=\mathcal{R}_{A}^{s} u+q u<0 \quad \text { in } \Omega .
$$

Proposition 3.6. Suppose $0<c \leq q(x) \in L^{\infty}(\Omega)$. If $u \in H^{s}\left(\mathbb{R}^{n}\right)$ solves the exterior problem

$$
\left\{\begin{aligned}
\left(\mathcal{R}_{A}^{s}+q\right) u=f & \text { in } \Omega \\
u=g & \text { in } \Omega_{e}
\end{aligned}\right.
$$

for $f \in L^{\infty}(\Omega)$ and $g \in C_{c}^{\infty}\left(\Omega_{e}\right)$, then

$$
\|u\|_{L^{\infty}} \leq C\|f\|_{L^{\infty}(\Omega)}+\|g\|_{L^{\infty}\left(\Omega_{e}\right)} .
$$

Proof. Fix a function $\phi \in C_{c}^{\infty}\left(\mathbb{R}^{n}\right)$ s.t. $0 \leq \phi \leq 1$ and $\phi=1$ on $\bar{\Omega} \cup \operatorname{supp} g$.

It is clear from the pointwise definition of $\mathcal{R}_{A}^{s}$ that $\mathcal{R}_{A}^{s} \phi \geq 0$ in $\Omega$ so

$$
\left(\mathcal{R}_{A}^{s}+q\right) \phi \geq c \text { in } \Omega \text {. }
$$

Now let $\tilde{\phi}:=\left(\frac{1}{c}\|f\|_{L^{\infty}(\Omega)}+\|g\|_{L^{\infty}\left(\Omega_{e}\right)}\right) \phi$, then $\tilde{\phi} \pm u \geq 0$ in $\Omega_{e}$ and

$$
\left(\mathcal{R}_{A}^{s}+q\right)(\tilde{\phi} \pm u) \geq 0 \quad \text { in } \Omega
$$

so $|u| \leq \tilde{\phi}$ by the previous proposition.

\section{$3.2 \quad$ Hölder Regularity}

Proposition 3.7. (Proposition 1.1 in [2Q]) If $u \in \tilde{H}^{s}(\Omega)$ and $(-\Delta)^{s} u=f \in L^{\infty}(\Omega)$, then $u \in$ $C^{s}\left(\mathbb{R}^{n}\right)$ and

$$
\|u\|_{C^{s}\left(\mathbb{R}^{n}\right)} \leq C|| f \|_{L^{\infty}(\Omega)} .
$$

Based on the proposition above, we now prove the Hölder regularity theorem for the linear exterior problem (7).

Proposition 3.8. Suppose $\Omega \cup \operatorname{supp} A \subset B_{r}(0)$ for some $r>0$ and $\|A\|_{L^{\infty}\left(\mathbb{R}^{n}\right)} \leq \pi /(8 \sqrt{n} r)$, $0<c \leq q(x) \in L^{\infty}(\Omega)$ for some $c>0, W \cap B_{3 r}(0)=\emptyset$. If $g \in C_{c}^{\infty}(W)$, then $u=P_{A, q} g \in C^{s}\left(\mathbb{R}^{n}\right)$ where $P_{A, q}$ is solution operator associated with (7) and

$$
\|u\|_{C^{s}\left(\mathbb{R}^{n}\right)} \leq C\|g\|_{C^{2}\left(\mathbb{R}^{n}\right)} .
$$

Proof. Note that $v:=u-g \in \tilde{H}^{s}(\Omega)$ and

$$
(-\Delta)^{s} v=\left((-\Delta)^{s}-\mathcal{R}_{A}^{s}\right) v-\mathcal{R}_{A}^{s} g-q u \text { in } \Omega .
$$

By Proposition 3.6, $\|v\|_{L^{\infty}} \leq C_{1}\|g\|_{L^{\infty}}$. By Lemma 3.4,

$$
\left\|\left((-\Delta)^{s}-\mathcal{R}_{A}^{s}\right) v\right\|_{L^{\infty}\left(\mathbb{R}^{n}\right)} \leq C_{2}\|v\|_{L^{\infty}} \leq C_{3}\|g\|_{L^{\infty}} .
$$


Since $W \cap B_{3 r}(0)=\emptyset$, then $\left|\frac{x+y}{2}\right| \geq r, R_{A}(x, y)=1$ for $x \in \Omega, y \in W$ so

$$
\mathcal{R}_{A}^{s} g=(-\Delta)^{s} g \text { in } \Omega .
$$

By Lemma 3.3, $\left\|\mathcal{R}_{A}^{s} g\right\|_{L^{\infty}(\Omega)} \leq C^{\prime}\|g\|_{C^{2}\left(\mathbb{R}^{n}\right)}$. Hence

$$
\left\|\left((-\Delta)^{s}-\mathcal{R}_{A}^{s}\right) v-\mathcal{R}_{A}^{s} g-q u\right\|_{L^{\infty}(\Omega)} \leq C^{\prime \prime}\|g\|_{C^{2}\left(\mathbb{R}^{n}\right)}
$$

Now by the proposition above, we have $\|v\|_{C^{s}\left(\mathbb{R}^{n}\right)} \leq C\|g\|_{C^{2}\left(\mathbb{R}^{n}\right)}$.

\section{The Nonlinear Exterior Problem}

Now we consider the nonlinear exterior problem

$$
\left\{\begin{aligned}
\mathcal{R}_{A}^{s} u+a(x, u)=0 & & \text { in } \Omega \\
u=g & & \text { in } \Omega_{e} .
\end{aligned}\right.
$$

Recall that we assume the nonlinearity $a(\cdot, \cdot)$ has the Taylor's expansion (4) with coefficients $a_{k}(x) \in C^{s}(\Omega)$ and we also assume $a_{1}(x) \geq c>0$. We write

$$
R_{m}(x, z):=\sum_{k=m+1}^{\infty} \frac{a_{k}(x)}{k !} z^{k} .
$$

We note that $C^{s}(\Omega)$ is an algebra since for $u_{1}, u_{2} \in C^{s}(\Omega)$, we have

$$
\left\|u_{1} u_{2}\right\|_{C^{s}(\Omega)} \leq C_{0}\left(\left\|u_{1}\right\|_{C^{s}(\Omega)}\left\|u_{2}\right\|_{L^{\infty}(\Omega)}+\left\|u_{1}\right\|_{L^{\infty}(\Omega)}\left\|u_{2}\right\|_{C^{s}(\Omega)}\right)
$$

(see Theorem A.7 in [1]) so

$$
\left\|u_{1} u_{2}\right\|_{C^{s}(\Omega)} \leq 2 C_{0}\left\|u_{1}\right\|_{C^{s}(\Omega)}\left\|u_{2}\right\|_{C^{s}(\Omega)} .
$$

Also note that by Cauchy's estimate, we have

$$
\left\|a_{k}\right\|_{C^{s}(\Omega)} \leq \frac{k !}{R^{k}} \sup _{z \in \mathbb{C},|z|=R}\|a(\cdot, z)\|_{C^{s}(\Omega)}, \quad R>0 .
$$

Based on the estimates above, we have the following estimates when we choose $R=\max \left\{4 C_{0}, 1\right\}$.

Proposition 4.1. If $\|u\|_{C^{s}(\Omega)} \leq 1$, then

$$
\begin{aligned}
\sum_{k=m+1}^{\infty}\left\|\frac{a_{k}(x)}{k !} u^{k}\right\|_{C^{s}(\Omega)} & \leq\left(\sum_{k=m+1}^{\infty} \frac{1}{2^{k}}\right) \sup _{z \in \mathbb{C},|z|=R}\|a(\cdot, z)\|_{C^{s}(\Omega)}\|u\|_{C^{s}(\Omega)}^{m+1}, \\
\sum_{k=m+1}^{\infty}\left\|\frac{a_{k}(x)}{(k-1) !} u^{k-1}\right\|_{C^{s}(\Omega)} & \leq\left(\sum_{k=m+1}^{\infty} \frac{k}{2^{k-1}}\right) \sup _{z \in \mathbb{C},|z|=R}\|a(\cdot, z)\|_{C^{s}(\Omega)}\|u\|_{C^{s}(\Omega)}^{m} .
\end{aligned}
$$

The following proposition is an analogue of Theorem 2.1 in [18]. 
Proposition 4.2. Suppose $\Omega \cup \operatorname{supp} A \subset B_{r}(0)$ for some $r>0$ and $\|A\|_{L^{\infty}\left(\mathbb{R}^{n}\right)} \leq \pi /(8 \sqrt{n} r)$, $W \cap B_{3 r}(0)=\emptyset$ and $g \in C_{c}^{\infty}(W)$. There exists a small constant $\rho>0$ s.t. if $\|g\|_{C^{2}\left(\mathbb{R}^{n}\right)} \leq \rho$, then the nonlinear exterior problem (10) has a unique solution $u \in H^{s}\left(\mathbb{R}^{n}\right) \cap C^{s}\left(\mathbb{R}^{n}\right)$ satisfying

$$
\left(u-P_{A, a_{1}} g\right) \in M:=\left\{v \in C^{s}\left(\mathbb{R}^{n}\right):\left.v\right|_{\Omega_{e}}=0,\|v\|_{C^{s}\left(\mathbb{R}^{n}\right)} \leq \rho\right\} .
$$

Moreover, we have

$$
\|u\|_{C^{s}\left(\mathbb{R}^{n}\right)} \leq C\|g\|_{C^{2}\left(\mathbb{R}^{n}\right)} .
$$

Proof. Let $u_{0}:=P_{A, a_{1}} g$, then by Proposition 3.8 we have

$$
\left\|u_{0}\right\|_{C^{s}\left(\mathbb{R}^{n}\right)} \leq C_{1}\|g\|_{C^{2}\left(\mathbb{R}^{n}\right)}
$$

and the nonlinear exterior problem (10) can be written as

$$
\left\{\begin{aligned}
\mathcal{R}_{A}^{s}\left(u-u_{0}\right)+a_{1}(x)\left(u-u_{0}\right) & =-R_{1}(x, u) \quad \text { in } \Omega \\
u-u_{0} & =0 \quad \text { in } \Omega_{e} .
\end{aligned}\right.
$$

Now for $f \in L^{\infty}(\Omega)$, we consider the solution operator $J: f \rightarrow u_{f} \in \tilde{H}^{s}(\Omega)$ of

$$
\mathcal{R}_{A}^{s} u+a_{1}(x) u=f \quad \text { in } \Omega .
$$

We write

$$
(-\Delta)^{s} u=\left((-\Delta)^{s}-\mathcal{R}_{A}^{s}\right) u-a_{1}(x) u+f \quad \text { in } \Omega,
$$

so by Lemma 3.4, Proposition 3.6 and 3.7, we have $J(f) \in C^{s}\left(\mathbb{R}^{n}\right)$ and

$$
\|J(f)\|_{C^{s}\left(\mathbb{R}^{n}\right)} \leq C_{2}\|f\|_{L^{\infty}(\Omega)} .
$$

We define maps $G, F$ by

$$
G(v):=R_{1}\left(x, u_{0}+v\right), \quad F:=J \circ G .
$$

We will show that $F$ is a contraction map on the complete metric space $M$ for small $\rho$, which will be chosen later. In fact, for small $\rho$ and $v \in M$, we have

$$
\|F(v)\|_{C^{s}\left(\mathbb{R}^{n}\right)} \leq C_{2}\|G(v)\|_{L^{\infty}(\Omega)}=C_{2}\left\|R_{1}\left(x, u_{0}+v\right)\right\|_{L^{\infty}(\Omega)} \leq C_{2}^{\prime}\left\|u_{0}+v\right\|_{C^{s}(\Omega)}^{2} \leq C_{2}^{\prime \prime} \rho^{2} .
$$

Here we use the first estimate in Proposition 4.1 and the constant $C_{2}^{\prime \prime}$ is independent of $\rho$.

Also for small $\rho$ and $v_{1}, v_{2} \in M$, we have

$$
\begin{gathered}
\left\|F\left(v_{1}\right)-F\left(v_{2}\right)\right\|_{C^{s}\left(\mathbb{R}^{n}\right)} \leq C_{2}\left\|G\left(v_{1}\right)-G\left(v_{2}\right)\right\|_{L^{\infty}(\Omega)} \\
=C_{2}\left\|R_{1}\left(x, u_{0}+v_{1}\right)-R_{1}\left(x, u_{0}+v_{2}\right)\right\|_{L^{\infty}(\Omega)} \\
\leq\left.\left\|v_{1}-v_{2}\right\|\right|_{L^{\infty}(\Omega)} \sum_{k=2}^{\infty}\left\|\frac{a_{k}(x)}{(k-1) !}\left(\left|u_{0}+v_{1}\right|^{k-1}+\left|u_{0}+v_{1}\right|^{k-1}\right)\right\|_{L^{\infty}(\Omega)} \\
\leq C_{3}\left\|v_{1}-v_{2}\right\|_{L^{\infty}(\Omega)}\left(\left\|u_{0}+v_{1}\right\|_{C^{s}(\Omega)}+\left\|u_{0}+v_{2}\right\|_{C^{s}(\Omega)}\right) \\
\leq C_{4} \rho\left\|v_{1}-v_{2}\right\|_{L^{\infty}(\Omega)} .
\end{gathered}
$$


Here we use the inequality

$$
\left|a^{m}-b^{m}\right| \leq m|a-b|\left(|a|^{m-1}+|b|^{m-1}\right)
$$

and the second estimate in Proposition 4.1. The constant $C_{4}$ is independent of $\rho$.

Hence, for small $\rho<1 /\left(C_{2}^{\prime \prime}+C_{4}\right), F$ is a contraction map on $M$ so by Banach fixed-point theorem, there exists a unique $v_{0} \in M$ s.t. $F\left(v_{0}\right)=v_{0}$.

Now note that $v_{0}=F\left(v_{0}\right) \in \tilde{H}^{s}(\Omega)$ and

$$
\left\|v_{0}\right\|_{C^{s}\left(\mathbb{R}^{n}\right)}=\left\|F\left(v_{0}\right)\right\|_{C^{s}\left(\mathbb{R}^{n}\right)} \leq C_{2}^{\prime}\left\|u_{0}+v_{0}\right\|_{C^{s}(\Omega)}^{2} \leq C_{3}^{\prime} \rho\left(\left\|u_{0}\right\|_{C^{s}(\Omega)}+\left\|v_{0}\right\|_{C^{s}(\Omega)}\right)
$$

where the constant $C_{3}^{\prime}$ is independent of $\rho$. Hence, for small $\rho<1 /\left(2 C_{3}^{\prime}\right)$, we have

$$
\left\|v_{0}\right\|_{C^{s}\left(\mathbb{R}^{n}\right)} \leq 2 C_{3}^{\prime} \rho\left\|u_{0}\right\|_{C^{s}\left(\mathbb{R}^{n}\right)}
$$

and then $u:=u_{0}+v_{0}$ satisfies

$$
\|u\|_{C^{s}\left(\mathbb{R}^{n}\right)} \leq C\|g\|_{C^{2}\left(\mathbb{R}^{n}\right)} .
$$

\section{The Inverse Problem}

From now on, we denote the bounded solution operator associated with (10) by $Q_{A, a}$.

Proposition 4.2 ensures that the Dirichlet-to-Neumann map $\Lambda_{A, a}$ given by (5) is well-defined for $g$ satisfying the condition assumed in the statement of the proposition. We remark that $\Lambda_{A, a}$ coincides the Dirichlet-to-Neumann map defined in Subsection 2.3 in 18 when $A=0$.

The first order linearization in $H^{s}\left(\mathbb{R}^{n}\right)$ will be useful when we prove Theorem 1.1 later.

Proposition 5.1. Suppose $\Omega \cup \operatorname{supp} A \subset B_{r}(0)$ for some $r>0$ and $\|A\|_{L^{\infty}\left(\mathbb{R}^{n}\right)} \leq \pi /(8 \sqrt{n} r)$, $W \cap B_{3 r}(0)=\emptyset$ and $g \in C_{c}^{\infty}(W)$, then

$$
Q_{A, a}(\epsilon g) / \epsilon \rightarrow P_{A, a_{1}} g
$$

in $H^{s}\left(\mathbb{R}^{n}\right)$ as $\epsilon \rightarrow 0$.

Proof. Write $u_{\epsilon, g}:=Q_{A, a}(\epsilon g)$ and $u_{g}:=P_{A, a_{1}} g$ for sufficiently small $\epsilon$.

Note that $v_{\epsilon, g}:=u_{g}-\frac{u_{\epsilon, g}}{\epsilon} \in \tilde{H}^{s}(\Omega)$ and we have

$$
\mathcal{R}_{A}^{s} v_{\epsilon, g}+a_{1}(x) v_{\epsilon, g}=\frac{1}{\epsilon} R_{1}\left(x, u_{\epsilon, g}\right) \quad \text { in } \Omega .
$$

Now choose $v_{\epsilon, g}$ as a test function, then by Proposition 2.3 we have

$$
\left\langle\mathcal{R}_{A}^{s} v_{\epsilon, g}+a_{1} v_{\epsilon, g}, v_{\epsilon, g}\right\rangle \geq\left[v_{\epsilon, g}\right]_{H_{A}^{s}}^{2}+c\left\|v_{\epsilon, g}\right\|_{L^{2}(\Omega)}^{2} \geq c^{\prime}\left\|v_{\epsilon, g}\right\|_{H^{s}}^{2}
$$

and by Proposition 4.1 and 4.2, we have

$$
\begin{aligned}
& \left|\left\langle\frac{1}{\epsilon} R_{1}\left(x, u_{\epsilon, g}\right), v_{\epsilon, g}\right\rangle\right| \leq \frac{C}{\epsilon}\left\|R_{1}\left(x, u_{\epsilon, g}\right)\right\|_{L^{\infty}(\Omega)}\left\|v_{\epsilon, g}\right\|_{L^{2}(\Omega)} \\
\leq & \frac{C^{\prime}}{\epsilon}\left\|u_{\epsilon, g}\right\|_{C^{s}\left(\mathbb{R}^{n}\right)}^{2}\left\|v_{\epsilon, g}\right\|_{L^{2}(\Omega)} \leq C^{\prime \prime} \epsilon\|g\|_{C^{2}\left(\mathbb{R}^{n}\right)}^{2}\left\|v_{\epsilon, g}\right\|_{L^{2}(\Omega)} .
\end{aligned}
$$

Hence we have

$$
\left\|v_{\epsilon, g}\right\|_{H^{s}} \leq C^{\prime \prime \prime} \epsilon\|g\|_{C^{2}\left(\mathbb{R}^{n}\right)}^{2} .
$$

Now it is clear that $v_{\epsilon, g} \rightarrow 0$ in $H^{s}\left(\mathbb{R}^{n}\right)$ as $\epsilon \rightarrow 0$. 
Now we are ready to prove Theorem 1.1.

Proof. Write $u_{\epsilon, g}^{(j)}:=Q_{A, a^{(j)}}(\epsilon g)$ and $u_{g}^{(j)}:=P_{A, a_{1}^{(j)}}(g)$ for $g \in C_{c}^{\infty}\left(W_{1}\right)$ and sufficiently small $\epsilon$.

By the assumption, we have

$$
\mathcal{R}_{A}^{s} u_{\epsilon, g}^{(1)}=\mathcal{R}_{A}^{s} u_{\epsilon, g}^{(2)} \quad \text { in } W_{2} .
$$

Since $u_{\epsilon, g}^{(1)}=u_{\epsilon, g}^{(2)}=\epsilon g$ in $\Omega_{e}$, then by Proposition 2.4 we have $u_{\epsilon, g}^{(1)}=u_{\epsilon, g}^{(2)}=: u_{\epsilon, g}$ in $\mathbb{R}^{n}$ so

$$
\mathcal{R}_{A}^{s} u_{\epsilon, g}+a^{(j)}\left(x, u_{\epsilon, g}\right)=0 \quad \text { in } \Omega \quad(j=1,2),
$$

which implies

$$
\left(a_{1}^{(1)}(x)-a_{1}^{(2)}(x)\right) u_{\epsilon, g}=R_{1}^{(2)}\left(x, u_{\epsilon, g}\right)-R_{1}^{(1)}\left(x, u_{\epsilon, g}\right) \quad \text { in } \Omega .
$$

Now note that

$$
\begin{gathered}
\left\|a_{1}^{(1)}(x)-a_{1}^{(2)}(x)\right\|_{L^{2}(\Omega)} \leq\left\|\left(a_{1}^{(1)}(x)-a_{1}^{(2)}(x)\right)\left(1-\frac{u_{\epsilon, g}}{\epsilon}\right)\right\|_{L^{2}(\Omega)}+\frac{1}{\epsilon}\left\|\left(a_{1}^{(1)}(x)-a_{1}^{(2)}(x)\right) u_{\epsilon, g}\right\|_{L^{2}(\Omega)} \\
\leq\left\|a_{1}^{(1)}(x)-a_{1}^{(2)}(x)\right\|_{L^{\infty}(\Omega)}\left\|1-\frac{u_{\epsilon, g}}{\epsilon}\right\|_{L^{2}(\Omega)}+\frac{1}{\epsilon}\left\|\left(a_{1}^{(1)}(x)-a_{1}^{(2)}(x)\right) u_{\epsilon, g}\right\|_{L^{2}(\Omega)}
\end{gathered}
$$

For given $\delta>0$, by Proposition 3.2 we can choose $g \in C_{c}^{\infty}\left(W_{1}\right)$ s.t.

$$
\left\|1-u_{g}\right\|_{L^{2}(\Omega)} \leq \delta
$$

For this chosen $g$, we have

$$
\left\|1-\frac{u_{\epsilon, g}}{\epsilon}\right\|_{L^{2}(\Omega)} \leq 2 \delta
$$

for small $\epsilon$ by Proposition 5.1 and we also have

$$
\begin{gathered}
\frac{1}{\epsilon}\left\|\left(a_{1}^{(1)}(x)-a_{1}^{(2)}(x)\right) u_{\epsilon, g}\right\|_{L^{2}(\Omega)} \leq \frac{C}{\epsilon}\left\|R_{1}^{(2)}\left(x, u_{\epsilon, g}\right)-R_{1}^{(1)}\left(x, u_{\epsilon, g}\right)\right\|_{L^{\infty}(\Omega)} \\
\leq \frac{C^{\prime}}{\epsilon}\left\|u_{\epsilon, g}\right\|_{C^{s}(\Omega)}^{2} \leq C^{\prime \prime} \epsilon\|g\|_{C^{2}\left(\mathbb{R}^{n}\right)}^{2}
\end{gathered}
$$

for small $\epsilon$ by Proposition 4.1 and 4.2 .

Now let $\epsilon \rightarrow 0$ in (12), then we have

$$
\left\|a_{1}^{(1)}(x)-a_{1}^{(2)}(x)\right\|_{L^{2}(\Omega)} \leq 2 \delta\left\|a_{1}^{(1)}(x)-a_{1}^{(2)}(x)\right\|_{L^{\infty}(\Omega)} .
$$

Since $\delta$ is arbitrary, then $a_{1}^{(1)}=a_{1}^{(2)}=: a_{1}$.

Iteratively, once we have shown $a_{j}^{(1)}=a_{j}^{(2)}(1 \leq j \leq l-1)$, then we have

$$
\frac{1}{l !}\left(a_{l}^{(1)}(x)-a_{l}^{(2)}(x)\right) u_{\epsilon, g}^{l}=R_{l}^{(2)}\left(x, u_{\epsilon, g}\right)-R_{l}^{(1)}\left(x, u_{\epsilon, g}\right) \quad \text { in } \Omega .
$$

Now note that

$$
\begin{gathered}
\|\| a_{l}^{(1)}(x)-\left.a_{l}^{(2)}(x)\right|^{\frac{1}{l}} \|_{L^{2}(\Omega)} \\
\leq\left|\left\|a_{l}^{(1)}(x)-\left.a_{l}^{(2)}(x)\right|^{\frac{1}{l}}\right\|_{L^{\infty}(\Omega)}\left\|1-\frac{u_{\epsilon, g}}{\epsilon}\right\|_{L^{2}(\Omega)}+\frac{1}{\epsilon}\right|\left\|a_{l}^{(1)}(x)-\left.a_{l}^{(2)}(x)\right|^{\frac{1}{l}} u_{\epsilon, g}\right\|_{L^{2}(\Omega)} .
\end{gathered}
$$


For given $\delta>0$, we can choose $g \in C_{c}^{\infty}\left(W_{1}\right)$ s.t.

$$
\left\|1-u_{g}\right\|_{L^{2}(\Omega)} \leq \delta
$$

and for this chosen $g$

$$
\begin{gathered}
\frac{1}{\epsilon}\left\|\left|a_{l}^{(1)}(x)-a_{l}^{(2)}(x)\right|^{\frac{1}{\tau}} u_{\epsilon, g}\right\|_{L^{2}(\Omega)} \leq \frac{C}{\epsilon}\left\|R_{l}^{(2)}\left(x, u_{\epsilon, g}\right)-R_{l}^{(1)}\left(x, u_{\epsilon, g}\right)\right\|_{L^{\infty}(\Omega)}^{\frac{1}{T}} \\
\leq \frac{C^{\prime}}{\epsilon}\left\|u_{\epsilon, g}\right\|_{C^{s}(\Omega)}^{\frac{l+1}{+}} \leq C^{\prime \prime} \epsilon^{\frac{1}{T}}\|g\|_{C^{2}\left(\mathbb{R}^{n}\right)}^{\frac{l+1}{+}}
\end{gathered}
$$

for small $\epsilon$ by Proposition 4.1 and 4.2.

Now let $\epsilon \rightarrow 0$, then we have

$$
\left\||| a_{l}^{(1)}(x)-\left.a_{l}^{(2)}(x)\right|^{\frac{1}{l}}\right\|_{L^{2}(\Omega)} \leq 2 \delta\left|\left\|a_{l}^{(1)}(x)-\left.a_{l}^{(2)}(x)\right|^{\frac{1}{l}}\right\|_{L^{\infty}(\Omega)} .\right.
$$

Since $\delta$ is arbitrary, then $a_{l}^{(1)}=a_{l}^{(2)}$.

\section{References}

[1] Sombuddha Bhattacharyya, Tuhin Ghosh, and Gunther Uhlmann. Inverse problem for fractionallaplacian with lower order non-local perturbations. Transactions of the American Mathematical Society, 374:3053-3075, 2021.

[2] Xinlin Cao, Yi-Hsuan Lin, and Hongyu Liu. Simultaneously recovering potentials and embedded obstacles for anisotropic fractional Schrödinger operators. Inverse Problems and Imaging, 13:197-210, 2019 .

[3] Xinlin Cao and Hongyu Liu. Determining a fractional helmholtz system with unknown source and medium parameter. Communications in Mathematical Sciences, 17(7):1861-1876, 2019.

[4] Giovanni Covi. An inverse problem for the fractional Schrödinger equation in a magnetic field. Inverse Problems, 36(4):045004, 2020.

[5] Pietro d'Avenia and Marco Squassina. Ground states for fractional magnetic operators. ESAIM: Control, Optimisation and Calculus of Variations, 24(1):1-24, 2018.

[6] Eleonora Di Nezza, Giampiero Palatucci, and Enrico Valdinoci. Hitchhiker's guide to the fractional sobolev spaces. arXiv preprint arXiv:1104.4345, 2011.

[7] David Dos Santos Ferreira, Carlos E Kenig, Johannes Sjöstrand, and Gunther Uhlmann. Determining a magnetic Schrödinger operator from partial cauchy data. Communications in mathematical physics, 271(2):467-488, 2007.

[8] Tuhin Ghosh, Yi-Hsuan Lin, and Jingni Xiao. The Calderón problem for variable coefficients nonlocal elliptic operators. Communications in Partial Differential Equations, 42(12):1923-1961, 2017.

[9] Tuhin Ghosh, Angkana Rüland, Mikko Salo, and Gunther Uhlmann. Uniqueness and reconstruction for the fractional calderón problem with a single measurement. Journal of Functional Analysis, page 108505, 2020.

[10] Tuhin Ghosh, Mikko Salo, and Gunther Uhlmann. The Calderón problem for the fractional Schrödinger equation. Analysis \& PDE, 13(2):455-475, 2020.

[11] Lars Hormander. The boundary problems of physical geodesy. Archive for Rational Mechanics and Analysis, 62:1-52, 1976. 
[12] Victor Isakov and Adrian I Nachman. Global uniqueness for a two-dimensional semilinear elliptic inverse problem. Transactions of the American Mathematical Society, 347(9):3375-3390, 1995.

[13] Victor Isakov and John Sylvester. Global uniqueness for a semilinear elliptic inverse problem. Communications on Pure and Applied Mathematics, 47(10):1403-1410, 1994.

[14] Katsiaryna Krupchyk and Gunther Uhlmann. Uniqueness in an inverse boundary problem for a magnetic Schrödinger operator with a bounded magnetic potential. Communications in Mathematical Physics, 327(3):993-1009, 2014.

[15] Katya Krupchyk and Gunther Uhlmann. Partial data inverse problems for semilinear elliptic equations with gradient nonlinearities. Mathematical Research Letters, 27(6):1801-1824, 2020.

[16] Katya Krupchyk and Gunther Uhlmann. A remark on partial data inverse problems for semilinear elliptic equations. Proceedings of the American Mathematical Society, 148(2):681-685, 2020.

[17] Ru-Yu Lai and Yi-Hsuan Lin. Global uniqueness for the fractional semilinear Schrödinger equation. Proceedings of the American Mathematical Society, 147(3):1189-1199, 2019.

[18] Ru-Yu Lai and Yi-Hsuan Lin. Inverse problems for fractional semilinear elliptic equations. arXiv preprint arXiv:2004.00549, 2020.

[19] Matti Lassas, Tony Liimatainen, Yi-Hsuan Lin, and Mikko Salo. Inverse problems for elliptic equations with power type nonlinearities. Journal de mathématiques pures et appliquées, 145:44-82, 2021.

[20] Li Li. The Calderón problem for the fractional magnetic operator. Inverse Problems, 36(7):075003, 2020.

[21] Gen Nakamura, Ziqi Sun, and Gunther Uhlmann. Global identifiability for an inverse problem for the Schrödinger equation in a magnetic field. Mathematische Annalen, 303(1):377-388, 1995.

[22] Xavier Ros-Oton and Joaquim Serra. The dirichlet problem for the fractional laplacian: regularity up to the boundary. Journal de Mathématiques Pures et Appliquées, 101(3):275-302, 2014.

[23] Angkana Rüland and Mikko Salo. The fractional Calderón problem: low regularity and stability. Nonlinear Analysis, 193:111529, 2020.

[24] Marco Squassina and Bruno Volzone. Bourgain-Brézis-Mironescu formula for magnetic operators. Comptes Rendus Mathematique, 354(8):825-831, 2016.

[25] Zi Qi Sun. An inverse boundary value problem for Schrödinger operators with vector potentials. Transactions of the American Mathematical Society, 338(2):953-969, 1993.

[26] Ziqi Sun. An inverse boundary-value problem for semilinear elliptic equations. Electronic Journal of Differential Equations (EJDE)[electronic only], 2010:Paper-No, 2010.

[27] Ziqi Sun and Gunther Uhlmann. Inverse problems in quasilinear anisotropic media. American Journal of Mathematics, 119(4):771-797, 1997. 